\title{
Power-Law Distributions for the Citation Index of Scientific Publications and Scientists
}

\author{
Hari M. Gupta, José R. Campanha, and Rosana A. G. Pesce \\ Departamento de Física, Instituto de Geociências e Ciências Exatas, \\ UNESP, Caixa Postal 178, CEP 13500-970, Rio Claro, SP, Brazil
}

Received on 16 June, 2004. Revised version received on 15 September, 2005.

\begin{abstract}
The number of citations of a scientific publication or of an individual scientist has become an important factor of quality assessment in science. We report a study of the statistical distribution of the citation index of both scientific publications and scientists. We give numerical evidence that Tsallis (power law) statistics explains the entire distribution over eight orders of magnitude $\left(10^{-4}\right.$ to $\left.10^{4}\right)$. Also, we draw Zipf plots in order to analyze the statistical distribution of the citation index of Brazilian and international physicists and chemists. The relatively small group of Brazilian scientists seems more adequate to explain the dynamics of the citation index. In this case, we find that the distribution of the citation index can also be explained by a gradually truncated power law with similar parameters. We finally discuss possible mechanisms behind the citation index of scientists and scientific publications.
\end{abstract}

\section{INTRODUCTION}

In recent years physicists turned to the study of natural systems as a whole rather than in parts [1-6]. The difficulties in understanding these "complex systems" arise from the large number of elementary interactions that are taking place at the same time for a large number of components. Also, these systems are in constant evolution and do not have a usual equilibrium state [1]. Socio-economical and biological systems display these general features, and have been treated by physicists. Scaling power laws $[7,8]$ have been found in many biological [9-11], physical [2,12-20] and socio-economical systems [21-29], and they are now considered as an important property of these systems.

Scientific publications are a primary means of scholarly communication in science. The quality of a scientific paper or of an individual scientist can be gauged by the number of citations in the work of other authors. Although this cannot be an exact measurement of the relevance of either a paper or a scientist, it can be taken as a particular and reasonable measure. One of the problems of our scientific community is to know the mechanisms and the distribution of (i) the number of publications of a scientist, (ii) the number of citations, or citation index, of a scientific publication, and (iii) the citation index of a scientist.

In 1957, in a study of the publication record of the scientific research staff at Brookhaven National Laboratory, Shockley [30] claimed that the rate of scientific publications is described by a log-normal distribution. Laherrere and Sornette [31] presented numerical evidence, on the basis of data for the 1120 most cited physicists from 1981 to June 1997, that the citation distribution of individual authors is associated with a stretched exponential form, $N(x) \sim \exp \left[-\left(x / x_{0}\right)^{\beta}\right]$, with $\beta \simeq 0.3$. Using the technique of the Zipf plots, Redner [32] has recently shown that the distribution of citations of the most cited scientific papers is described by a power law, $N(x) \sim x^{-\alpha}$, with $\alpha \simeq 3.0$. Tsallis and Albuquerque [33] claim that the newly proposed "Tsallis statistics" can as well account for the distribution of citations of scientific papers.
The number of publications and the citation index are different concepts. The number of publications of a scientist represents the amount of work that he has done, while the citation index is much closer to representing the quality of this work. The number of publications depends on the capacity to work and to get papers published, while the citation index is related to factors as the originality, the interest in the community, and the relevance of particular research topics. The number of scientific works published by a scientist depends on some factors, as choosing a proper problem, working on this problem, choosing a proper journal, writing ability. As pointed by Schockley [30], a log-normal form is expected to account for the distribution of published scientific papers.

In the present paper we discuss the statistical distribution and the mechanisms behind the citation index of a scientific publication and of an individual scientist. In Section II, we present the model. In Section III, we analyze the statistical distribution of the citation index of scientific publications in 1981, which were cited between 1981 and June of 1997. We also analyze and compare the distributions of the citation index of highly cited Brazilian and international physicists and chemists. In Section IV, we discuss the results and possible mechanisms underlying the citation index.

\section{THE MODEL}

A power-law distribution $[7,8]$ has been first observed by Pareto in economics [8] in 1897. Pareto claimed that it was related to a positive feedback, namely that wealthy people can more efficiently level their wealth than the average individuals, so they can create more wealth and achieve an even higher level of income. Recently, we have related power-law distributions to effects of competition, learning and natural selection [34].

In the context of nonextensive thermodynamics, Tsallis $[35,36]$ was able to obtain power-law distributions with the inclusion of long-range interactions and long-range microscopic 
memory. The Tsallis generalized entropy is given by

$$
S=k \frac{1-\sum_{i} p_{i}^{q}}{q-1}
$$

where $k$ is a positive constant, $q$ is a parameter, and the sum is over the probabilities of the statistical states. On the basis of this definition, Tsallis and Albuquerque [4] derived the statistical distribution

$$
N(x)=\frac{N_{0}}{[1+(q-1) \lambda x]^{\frac{q}{q-1}}},
$$

where $N(x)$ is the probability density, $\lambda$ is a parameter, and $N_{0}$ is a normalization constant. This formula can also be written as

$$
N(x)=\frac{N_{0}}{\left[1+c_{1} x\right]^{(1+\alpha)}}
$$

where $c_{1}$ is a constant and $(1+\alpha)$ is a power-law index. For large values of $x$, this distribution becomes a simple power law,

$$
N(x) \approx c x^{-(1+\alpha)} .
$$

In this limit, $\log N(x)$ versus $\log x$ is just a straight line.

In real systems, power-law distributions cannot continue for ever. They have to be somehow truncated in order to avoid an infinite variance. For scientific publications, the research field becomes saturated or almost fully investigated after a certain time, which may be roughly taken between 20 to 100 years, depending on the particular field. Researchers in this area, and citations as well, begin to decrease after this period of saturation. In addition to the saturation of the field, there are human limitations to the production of a large number of relevant scientific works.

Recently, we have shown that, by gradually truncating a power-law distribution after a certain critical value, it is possible to explain the entire distribution including very large steps in financial and physical complex systems [37-39]. In this work, the power-law distributions come from a positive feedback which gradually ceases after a certain step size due to limited physical capacity of the components of the system or the system itself. In this limit, these distributions approach a normal form [37]. This approach may also lead to a distribution of the citation index, given by

$$
N(x)=c x^{-(1+\alpha)} f(x),
$$

with

$$
f(x)=\left\{\begin{array}{cl}
1 & \text { if }|x| \leqslant x_{c} \\
\exp \left\{-\left(\frac{\left(|x|-x_{c}\right)}{k}\right)^{\beta}\right\} & \text { if }|x|>x_{c}
\end{array},\right.
$$

where $x_{c}$ is the critical value of the step size at which the probability distribution begins to deviate from a power-law distribution due to physical limitations, and $k$ is related to the sharpness of the cut-off. Comparing to a normal distribution, we have

$$
\beta=2-\alpha
$$

We now consider two special cases:

(i) case I, if $x \leqslant x_{C}$, with

$$
N(x)=c x^{-(1+\alpha)},
$$

which gives a power-law distribution;

(ii) case II, if $x>>x_{C}$, in which the variation due to $f(x)$ is dominant, and we have

$$
N(x) \sim \exp \left\{-\left(\frac{|x|}{k}\right)^{\beta}\right\}
$$

so $\log N(x)$ versus $x^{\beta}$ is a straight line. This gives a stretched exponential distribution.

The publication density is usually very small for highly cited papers. It is then interesting to draw a Zipf plot [40], in which the number of citations of the $n t h$ most cited paper out of an ensemble of $M$ papers is plotted versus the rank $n$. By its very definition, the Zipf plot is closely related to the cumulative large- $x$ tail of the citation distribution, which makes it well suited for determining the large- $x$ tail of the citation distribution. Also, it smooths out the fluctuations in the highcitation tail and thus facilitates a quantitative analysis.

Given an ensemble of $M$ papers and the corresponding number of citations for each of these papers, according to the rank order, $Y_{1} \geqslant Y_{2} \geqslant Y_{3} \ldots \geqslant Y_{n} \geqslant \ldots Y_{M}$, the number of citations of the $n t h$ most cited paper $Y_{n}$ may be estimated from the criterion [24]

$$
\int_{Y_{n}}^{\infty} N(x) d x=\int_{Y_{n}}^{\infty} M \cdot P(x) d x=n .
$$

This equation means that there are $n$ out of the ensemble of $M$ papers which are cited at least $Y_{n}$ times. From the dependence of $Y_{n}$ with $n$ in a Zipf plot, we can test whether it agrees with a proposed form for $N(x)$.

For a simple power law distribution, using Equations (4) or (8), we obtain

$$
Y_{n}=c_{1} M \alpha n^{-\frac{1}{\alpha}}
$$

which can also be written as

$$
\log Y_{n}=\left(-\frac{1}{\alpha}\right) \log n+b,
$$

so $\log Y_{n}$ versus $\log n$ gives a straight line and $b$ is a constant.

For a stretched exponential distribution, using Equation (9), we obtain

$$
Y_{n}^{\beta}=-a \ln n+b,
$$

where $a$ and $b$ are constants. In this case, $Y_{n}^{\beta}$ versus $\ln n$ is a straight line.

\section{DATA ANALYSIS}

\section{A. Scientific Publications}

We now investigate one of the largest data sets of scientific publications, stored by the Institute for Scientific Information, 
ISI. It contains the distribution of citations of 783399 papers (with 6716198 citations) published in 1981 and cited between 1981 and June 1997 [41]. It includes papers published in all levels of journals. First, we consider the citations of publications. In both the earlier works on the citation of scientific publications [32,33], the number of publications versus citations is plotted and zeros are simply ignored. In a statistical distribution, we obtain the publication density, in other words, the number of publications in the interval of unit citation, instead of the number of publications. This makes a difference in the distribution of the most cited papers as we have a few papers in this range. Furthermore, the zeros can not be ignored as $\ln (0)$ is $-\infty$. In order to have a picture of the citation mechanisms, we then plot and discuss the publication density (the number of publications per citation) versus the number of citations.

The publication density is given by

$$
N(x)=\left[\frac{\Delta N}{\Delta x}\right]_{x},
$$

where $\Delta N$ is the number of publications with citations between $x-\Delta x / 2$ and $x+\Delta x / 2$. For smaller citation indices, as we have a large number of publications, we took $\Delta x=1$; for larger citation indices, we gradually increased $\Delta x$ in order to have just non-zero values of the publication density for citations.

We observe that for almost eight orders of magnitude $\left(10^{-4}\right.$ to $10^{4}$ ) of publication density, the distribution is given by Tsallis statistics, as shown in Fig. 1 , with $N_{0}=4.66 \times 10^{4}$, $c_{1}=0.058$ and $(1+\alpha)=3.1$. The values of $\mathrm{N}_{0}$ and $\alpha$ are chosen from the slope of the best straight-line fittting, with $x \geq 100 ; c_{1}$ is chosen to give a best fitting for the initial stages.

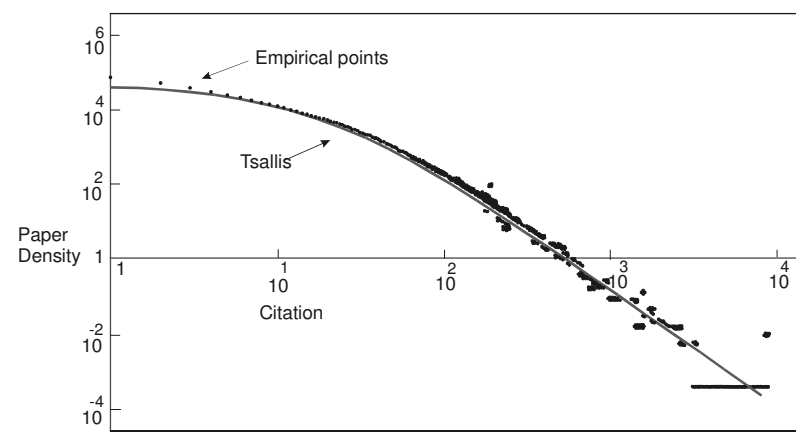

FIG. 1: Publication density (publications per citation) versus citation distribution for 783339 papers in the ISI data on a double logarithmic scale. The continuous line is a theoretical curve coming from Tsallis statistics, and the dots are empirical points.

In order to further confirm the supposed distribution, we draw Zipf plots in Fig. 2, for the same parameter values. The fit is excellent showing that the distribution can really be associated with a Tsallis (power-law) statistics. Also, the fits show that the stretched exponential distribution is not suitable for the present case.

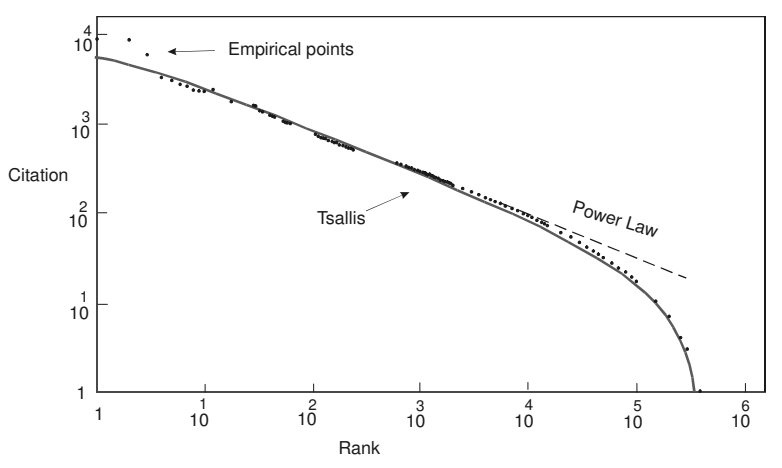

FIG. 2: Zipf plot of the number of citation of the $n t h$ ranked paper $Y_{n}$ versus rank $n$ on a double logarithmic scale of ISI data. The continuous line is a theoretical curve on the basis of Tsallis statistics, the dots are empirical points, and the dashes come from a power law.

\section{B. Scientists}

In this case, we analyze the citation index of (a) most cited Brazilian physicists and chemists and (b) most cited physicists and chemists in the world. All physicists (chemists), including Brazilian physicists, publish their work in the same journals and work on almost the same problems. The Brazilian physicists (chemists) form a small sub-group within the international physicists (chemists) community. The limiting factor for Brazilian physicists (chemists) is relevant for a few top ranking scientists only, since the citation index of only these scientists is above the critical value $x_{C}$ of a gradually truncated power-law distribution. For the rest of the most cited scientists, a power law is sufficient, and the index of this power law can be more precisely obtained. For the most cited international scientists, the limiting factor may be relevant since the citation index of almost all of these scientists is above the critical value $x_{C}$.

In Fig. 3, we plot citation number $Y_{n}$ versus rank $n$ for the first 205 Brazilian physicists in 1999 [42]. As it should be expected in case I, we observe a straight line for larger values of $n$, which begins to deviate for smaller values of $n$ $(n<20)$. The theoretical curve corresponds to a gradually truncated power law, with $\alpha=1.53, x_{C}=2000, k=1000$, and $c=2 \times 10^{6}$. There is a good agreement between this theoretical curve and the empirical results.

In Fig. 4, we plot citation number $Y_{n}$ versus rank $n$ for the 1120 most cited physicists from 1981 to June 1997 [43,32], and compare the plots with the theoretical curves with the same values of the parameters as used in Fig. 4. The constant $c$ has been changed from $2 \times 10^{6}$ to $1 \times 10^{9}$, as the total number of physicists is much larger in this case. It is reasonable to consider that the citations of Brazilian physicists are roughly $0.2 \%$ of the total number of citations. Again, we have a good agreement. Note that we are able to explain both distributions with the same values of the basic parameters. In the case of international scientists, almost all physicists have a citation index larger than $x_{C}$, and therefore we can use a stretched ex- 


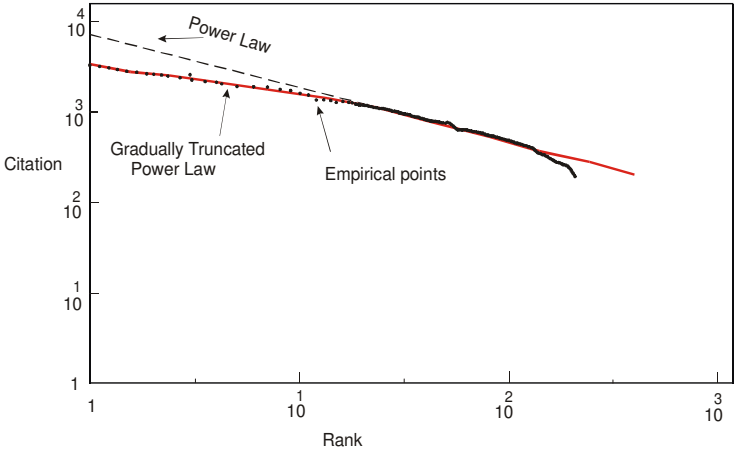

FIG. 3: Zipf plot of the number of citation of the $n t h$ ranked Brazilian physicist $Y_{n}$ versus rank $n$ on a double logarithmic scale. The dots are the empirical points, the straight line is a theoretical curve with a gradually truncated power law, and the dashes are a power law.

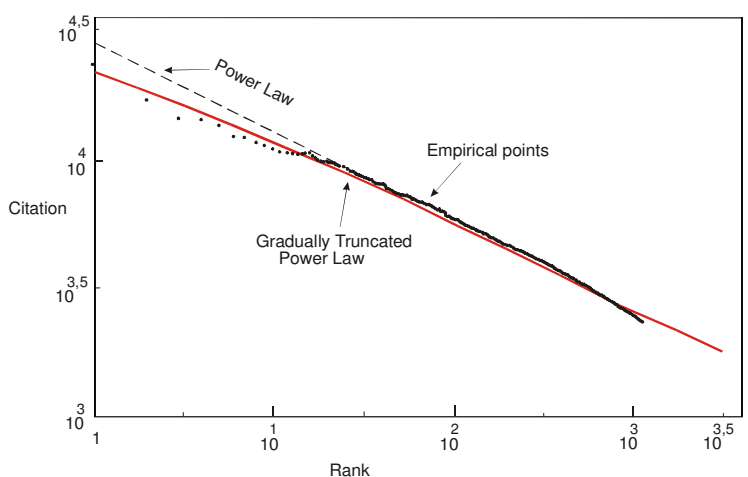

FIG. 4: Zipf plot of the number of citation of the $n t h$ ranked International physicist $Y_{n}$ versus rank n on a double logarithmic scale. The dots are the empirical points, the straight line is a theoretical curve with a gradually truncated power law using same parameters as in Figure 3, and the dashes are a power law.

ponential distribution as done by Laherrere and Sornette [31]. However, the stretched exponential distribution is not suitable for the citation index of Brazilian physicists. We then conclude that the distribution of the citation index of physicists is given by a "gradually truncated" power law.

In Fig. 5, we plot the citation number $Y_{n}$ versus rank $n$ for the first 119 Brazilian chemists in 1999 [42]. We see that there is a good agreement with a gradually truncated power law, with $\alpha=1.4, x_{C}=1500, k=2000$, and $c=2.5 \times 10^{5}$. In Fig. 6, we plot the citation number versus rank for the first 10858 chemists $[32,43]$ and compare this plot with a gradually truncated power law distribution with $\alpha=1.4, x_{C}=6000, k=$ 2000, and $c=10^{8}$. Again, there is a good agreement with the data.

The stretched exponential distribution fails for both international and Brazilian chemists. Although for physicists all the parameters are the same, $x_{C}$ for Brazilian chemists is much

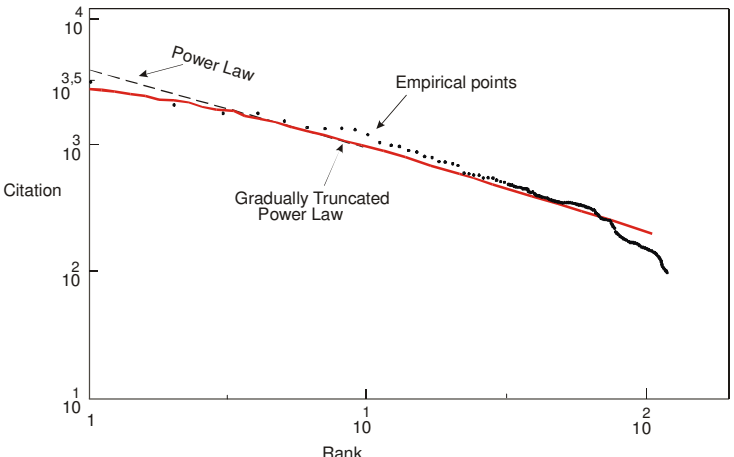

FIG. 5: Zipf plot of the number of citation of the $n t h$ ranked Brazilian chemist $Y_{n}$ versus rank $\mathrm{n}$ on a double logarithmic scale. The dots are the empirical data, the straight line is a theoretical curve with a gradually truncated power law distribution, and the dashes are a power-law distribution.

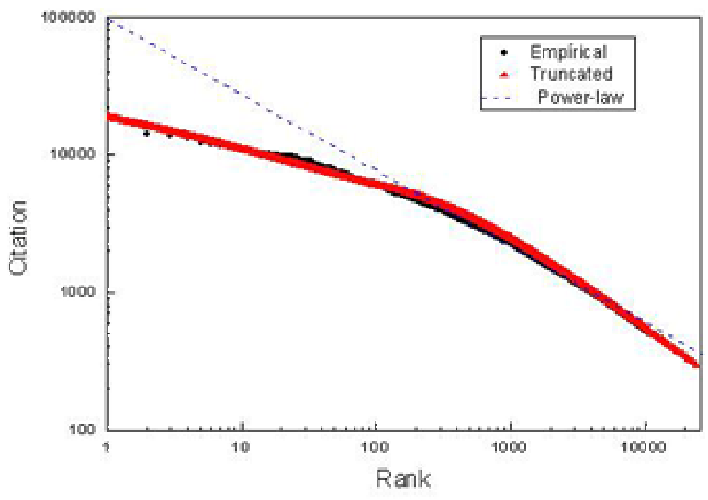

FIG. 6: Zipf plot of the number of citation of the nth ranked International chemist $Y_{n}$ versus rank $\mathrm{n}$ on a double logarithmic scale. The dots are the empirical data, the straight line is a theoretical curve with a gradually truncated power law, and the dashes are a power-law distribution.

lower than for international chemists. Perhaps this is due to more difficult procedures for obtaining financial support of research in Brazil, which is a more important factor in chemistry than in some branches of physics, particularly in theoretical physics. It is interesting to note that 8 out of the 10 most cited Brazilian physicists are working in theoretical physics.

\section{DISCUSSION}

It is usual that a scientific publication is first cited by people working in the same or closely related groups. Later on, it comes to the attention of other groups. In the second genera- 
Table I: Citations of the 20 most cited physicists from January 1981 to June 1997

\begin{tabular}{lllll} 
& \multicolumn{3}{l}{ \#cites/article \#articles \#cites } & rank \\
Witten, E. & 168.37 & 138 & 23235 & 1 \\
Gossard, A. C. & 40.56 & 419 & 16994 & 2 \\
Cava, R. J. & 64.60 & 223 & 14405 & 3 \\
Batlogg, B. & 83.32 & 170 & 141644 \\
Ploog, K. & 18.95 & 712 & 134915 \\
Ellis, J. & 40.18 & 305 & 12255 & 6 \\
Fisk, Z. & 23.13 & 520 & 120307 \\
Cardona, M. & 20.08 & 571 & 11465 & 8 \\
Nanopoulos, D. V. & 38.61 & 293 & 11314 & 9 \\
Heeger, A. J. & 33.98 & 320 & 10872 & 10 \\
Lee, P. A. & 72.89 & 146 & 10642 & 11 \\
Suzuki, T. & 7.58 & 1401 & 10617 & 12 \\
Anderson, P. W. & 80.30 & 138 & 10439 & 13 \\
Suzuki, M. & 11.60 & 898 & 10417 & 14 \\
Freeman, A. J. & 26.76 & 389 & 10411 & 15 \\
Tanaka, S. & 10.80 & 963 & 1040416 \\
Muller, K. A. & 82.37 & 122 & 1004917 \\
Schneemeyer, L. F. & 62.62 & 156 & 9768 & 18 \\
Chemla, D. S. & 59.68 & 162 & 9668 & 19 \\
Morkoc, H. & 20.27 & 477 & 9668 & 20
\end{tabular}

Table II: Citations of the 20 most cited chemists from January 1981 to June 1997

\begin{tabular}{lllll} 
& \multicolumn{3}{l}{ \#cites/article \#articles \#cites rank } \\
Bax, A. & 142.47 & 152 & 21655 & 1 \\
Pople, J. A. & 79.80 & 176 & 14044 & 2 \\
Scleyer, P. V. & 25.83 & 525 & 13559 & 3 \\
Ernst, R. R. & 71.81 & 182 & 13069 & 4 \\
Whitesides, G. M. 38.71 & 318 & 12310 & 5 \\
Schaefer, H. F. & 23.15 & 515 & 119216 \\
Huffman, J. C. & 20.20 & 577 & 11654 & 7 \\
Rheingold, A. L. & 13.63 & 830 & 11317 & 8 \\
Seebach, D. & 32.31 & 349 & 11275 & 9 \\
Lehn, J. M. & 35.25 & 307 & 10823 & 10 \\
Meyer, T. J. & 39.29 & 267 & 1049011 \\
Smalley, R. E. & 108.92 & 96 & 10456 & 12 \\
Bard, A. J. & 31.13 & 333 & 1036513 \\
Truhlar, D. G. & 31.43 & 328 & 1031014 \\
Stewart, J. J. P. & 261.00 & 39 & 10179 & 15 \\
Corey, E. J. & 33.43 & 303 & 1012916 \\
Yamamoto, Y. & 10.70 & 935 & 10007 & 17 \\
Tanaka, T. & 10.44 & 954 & 9961 & 18 \\
Cotton, F. A. & 15.63 & 634 & 9911 & 19 \\
Tanaka, K. & 8.17 & 1202 & 9820 & 20
\end{tabular}

tion of citations, it comes to the attention of other researchers through its citations. A more relevant paper is initially cited more times, comes to attention of more workers, and the number of citations increases rapidly. On the other hand, articles which are initially less cited come to attention of a few workers only, and their number of citations decreases very rapidly. Most of the published papers are forgotten in the first five years from publication. Only a few more relevant papers are cited during a very long time. A more cited paper in the initial stages comes to the attention of more workers, and thus is cited more times, thereby coming to the attention of still more people, and being cited still more times, and so on. The citation index increases much more rapidly for relevant papers, which leads to a power-law distribution.

A Zipf plot indicates that the first three most cited papers of a scientist are cited more times than it should be anticipated from a power-law distribution. The citation index of only the most cited papers is expected to increase with time, because other papers are more or less forgotten in twenty years. The presence of high-ranking outliers is often called the King effect and may be due to an amplifying process [44,45].

We basically agree with the conclusion of Tsallis and Albuquerque [4], with a slightly different power index (3.1 instead of 2.9). Note that we plot publication density, and that we achieved a good agreement over a much wider range, which leads to a much clearer picture of the distribution of the citation index.

The fact that a scientist is cited more times makes it easier to obtain financial support for his research projects and better students, which in turn contributes to form a better and larger group. A more cited scientist can more efficiently level his citation index than the average scientist, producing more citations and achieving higher levels of citation index. This positive feedback effect decreases gradually after a certain step size due to physical limitations of the system. For scientific publications, the citation index decreases with time after the field gets saturated or almost fully investigated, which normally takes 20 to 100 years depending on the field. As we have the citation index for a period of only 16 years, we have not observed any gradual truncation of a power law.

Apart from the saturation of the field, other limitations for scientists come from the human capacity to work. The citation index of a scientist is a product of the number of his articles and the average citation per article. It is not possible for any one to compete both in quality and quantity. Some people may have a large number of articles but a small average citation per article, while other people may have larger average citation of an article but small number of articles. This is clear in Tables I and II, in which we list the number of articles and the average citation per article of twenty internationally highly cited physicists and chemists. It is interesting to note that only two of them (P. W. Anderson, and K. A. Muller, at the 13th and 17 th places, respectively), out of the 20 most cited physicists, and six (J. A. Pople, R. R. Ernst, J. M. Lehn, R. E. Smalley, E. J. Corey, and K. Tanaka, at the 2nd, 4th, 10th, 12th, 16th, and 20th places, respectively), out of the 20 most cited chemists, are Nobel laureates.

As the citation index of only the most cited scientists is available, it is not possible to distinguish between Tsallis and power-law distributions. We then conclude that the distribution of the citation index of scientific publications and scientists is well represented by a gradually truncated powerlaw distribution. As in several systems from economy and physics, there is a positive feedback mechanism associated with this power law.

\section{Acknowledgments}

We are thankful to the anonymous referee for useful sug- 
gestions.

[1] P. Bak, How Nature Works, Oxford University Press, Oxford (1997)

[2] B. B. Mandelbrot, The Fractal Geometry of Nature (Freeman, New York 1982)

[3] B. B. Mandelbrot, Science 156, 637 (1967)

[4] D. L. Ruderman, Network: Computation in Neural Systems, 5, 517 (1994)

[5] L. Poon, C. Grebogi, Phys. Rev. Lett. 75, 4023 (1995)

[6] R. Badii, A. Politi, Complexity, Hierarchical Structures and Scaling in Physics, Cambridge, U. K. (1997)

[7] P. Lévy, Théorie de l'Addition des Variables Aléatories (Gauthier-Villars, Paris 1937)

[8] V. Pareto, Cours d'Economie Politique. Reprinted as a volume of Oeuvres Complètes (Droz, Geneve 1896-1965)

[9] C. K. Peng et al., Phys. Rev. Lett. 70, 1343 (1993)

[10] G. F. Zebende, P. M. C. de Oliveira, and T. J. Penna, Phys. Rev. E 57, 3311 (1998)

[11] J. B. Bassingthwaighte, L. S. Liebovitch, and B. J. West, Fractal Physiology (Oxford Univ. Press, New York 1994)

[12] U. Frish, M. F. Shlesinger, and G. Zaslavasky, Lévy Flights and Related Phenomena in Physics (Springer-Verlag, Berlin 1994)

[13] T. H. Solomon, E. R. Weeks, and H. L. Swinney, Phys. Rev. Lett. 71, 3975 (1993)

[14] M. Nelkin, Adv. Phys. 43, 143 (1994)

[15] A. Ott., J. P. Bouchard, D. Langevin, and W. Urbach, Phys. Rev. Lett. 65, 2201 (1990)

[16] Z. Olami, H. J. S. Feder, and K. Christensen, Phys. Rev. Lett. 68, 1244 (1992)

[17] B. Chabaud et. al., Phys. Rev. Lett. 73, 3227 (1994)

[18] E. Weeks, J. Urbach, and H. L. Swinney, Physica D97, 291 (1996)

[19] T. H. Solomon, E. R. Weeks, and H. L. Swinney, Physica D 76, 70 (1994)

[20] H. E. Hurst, Trans. Am. Soc. Civil Eng. 116, 770 (1951)

[21] Proceedings of First International Conference on High Fre- quency Data in Finance (Olsen \& Associates, Zurich 1995)

[22] R. N. Mantegna and H. E. Stanley, Nature 376, 46 (1995)

[23] G. Ghashghaie et. al., Nature 381, 767 (1996); A. Arneodo et al. preprint cond-mat/9607120

[24] E. F. Fama, Management Sci. 11, 404 (1965)

[25] A. L. Tucker, J. of Business \& Economic Statistics, 10, 73 (1992)

[26] J. P. Bouchaud and M. Potters, Théorie des risques financiers, Alea Saclay (1997)

[27] A. Arneodo, J. F. Muzy, and D. Sornette, Eur. Phys. J. B 2, 227 (1998)

[28] T. Lux and M. Marches, Nature 397, 498 (1999)

[29] H. M. Gupta , J. R. Campanha, and F. R. Chavarette, Int. J. Modern Phys. C 14, 449 (2003)

[30] W. Shockley, Proc IRE 45, 279 (1957)

[31] J. Laherrere, D. Sornette, Eur. Phys. J. B 13, 777 (2000)

[32] S. Redner, Eur. Phys. J. B 4, 131 (1998)

[33] C. Tsallis, M. P. Albuquerque, Eur. Phys. J. B 13, 777 (2000)

[34] H. M. Gupta, J. R. Campanha, Physica A 345, 267 (2005)

[35] C. Tsallis, J. Stat. Phys. 52, 479 (1988)

[36] C. Tsallis, Braz. J. Phys. 29, 1 (1999)

[37] H. M. Gupta, J. R. Campanha, Physica A 275, 531 (2000)

[38] H. M. Gupta, J. R. Campanha, Physica A 268, 231 (1999)

[39] H. M. Gupta, J. R. Campanha, and F. D. Prado, Int. Journal of Modern Physics C, 11, 1273 (2000)

[40] J. Galambos, The Assymptotic Theory of Extreme Order Statistics (John Wiley \& Sons, New York, 1978)

[41] Data from Web site http://physics.bu.edu/rredner/

[42] Data avalaible on website http://www.uol.com.br/fsp/especial/ranking

[43] Data avalaible on website http://physics.bu.edu/ redner/

[44] J. A. Davies, Eur. Phys. J. B 27, 445 (2002)

[45] D. Sornette, Proc. Natl. Acad. Sci. USA 99, 2522 (2002) 